\title{
Food addiction: un concetto di scarsa validità e utilità clinica
}

\author{
Riccardo Dalle Grave \\ Department of Eating and Weight Disorders, Villa Garda Hospital, Garda (VR)
}

\section{Parole chiave}

\section{Food addiction}

Obesità

Disturbi dell'alimentazione

Trattamento

Terapia cognitivo comportamentale

\begin{abstract}
Riassunto
Il concetto di food addiction ha attratto un crescente interesse nei media e ha stimolato molti dibattiti nella comunità scientifica. Scopo di questo articolo è analizzare la validità e l'utilità clinica del modello del food addiction applicato in particolare ai disturbi dell'alimentazione e all'obesità. Sebbene esistano alcune similitudini tra gli episodi di abbuffata riportati dalle persone con disturbi dell'alimentazione e il disturbo da uso di sostanze, esistono fondamentali differenze tra i due disturbi che riguardano la psicopatologia, l'epidemiologia e i fattori di rischio. Nelle persone con obesità, sebbene alcuni studi abbiano rivelato elementi comuni nei processi di ricompensa cerebrale tra l'assunzione di alcuni alimenti e l'uso di sostanze, questo non significa che un alimento in grado di attivare il sistema della ricompensa possa essere classificato come sostanza che crea dipendenza. L'alimentazione in eccesso riportata da alcune persone con obesità può, infatti, essere spiegata attraverso l'acquisizione di abitudini determinate dall'attivazione di processi edonici normali (non patologici), in un ambiente alimentare denso di energia e culturalmente permissivo. Inoltre, tra l'obesità e il disturbo da uso di sostanze esistono importanti differenze, come il decorso temporale della ricaduta e gli esiti del trattamento. Infine, l'adozione di un trattamento basato sul modello della food addiction nei disturbi dell'alimentazione e dell'obesità è fonte di preoccupazioni perché potrebbe allontanare le persone affette da questi disturbi da terapie di provata efficacia.
\end{abstract}

\section{Key words}

Food addiction

Obesity

Eating disorders

Treatment

Cognitive behavior therapy

\begin{abstract}
The concept of food addiction has attracted a growing interest in the media and has stimulated many debates in the scientific community. The aim of this article is to analyze the validity and clinical utility of the food addiction model applied to eating disorders and obesity, in particular. Although there are some similarities between the binge-eating episodes of reported by people with eating disorders and substance use disorder, there are fundamental differences between the two disorders concerning the psychopathology, epidemiology and risk factors. In people with obesity, although some studies have reported common brain reward processes between the intake of certain foods and the use of addictive substances, this does not mean that a food capable of activating the reward system can be classified as an addictive. Indeed, the overeating reported by some people with obesity may be explained through the acquisition of habits determined by the activation of normal hedonic processes (not pathological), in a food environment that is dense of energy and culturally permissive. Furthermore, there are important differences between obesity and substance use disorder, such as the time course of relapse and treatment outcomes. Finally, the adoption of a treatment based on the model of food addiction in eating disorders and obesity is a source of concern because it could move away the people suffering from these disorders from therapies of proven efficacy.
\end{abstract}

Copyright @ 2019 Riccardo Dalle Grave. This is an open-access article distributed under the terms of the Creative Commons Attribution License (CC BY). The use, distribution or reproduction in other forums is permitted, provided the original author(s) and the copyright owner(s) are credited and that the original publication in this journal is cited, in accordance with accepted academic practice. No use, distribution or reproduction is permitted which does not comply with these terms.

Riccardo Dalle Grave, MD (凶) rdalleg@gmail.com

Ricevuto: 30 Marzo 2019; Accettato: 15 Aprile 2019; Pubblicato online: 20 Aprile 2019. doi:10.32044/ijedo.2019.05 


\section{Introduzione}

Il concetto di food addiction ha attratto un crescente interesse nei media e ha stimolato molti dibattiti nella comunità scientifica. Scopo di questo articolo è analizzare la validità e l'utilità clinica del modello del food addiction applicato in particolare ai disturbi dell'alimentazione e all'obesità. Sebbene esistano alcune similitudini tra gli episodi di abbuffata riportati dalle persone con disturbi dell'alimentazione e il disturbo da uso di sostanze, esistono fondamentali differenze tra i due disturbi che riguardano la psicopatologia, l'epidemiologia e i fattori di rischio. Nelle persone con obesità, sebbene alcuni studi abbiano rivelato elementi comuni nei processi di ricompensa cerebrale tra l'assunzione di alcuni alimenti e l'uso di sostanze, questo non significa che un alimento in grado di attivare il sistema della ricompensa possa essere classificato come sostanza che crea dipendenza. L'alimentazione in eccesso riportata da alcune persone con obesità può, infatti, essere spiegata attraverso l'acquisizione di abitudini determinate dall'attivazione di processi edonici normali (non patologici), in un ambiente alimentare denso di energia e culturalmente permissivo. Inoltre, tra l'obesità e il disturbo da uso di sostanze esistono importanti differenze, come il decorso temporale della ricaduta e gli esiti del trattamento. Infine, l'adozione di un trattamento basato sul modello della food addiction nei disturbi dell'alimentazione e dell'obesità è fonte di preoccupazioni perché potrebbe allontanare le persone affette da questi disturbi da terapie di provata efficacia.

\section{Food addiction}

I primi resoconti scientifici che hanno usato il termine "addiction" facendo riferimento al cibo, e in particolare alla cioccolata, risalgono al 1890 (Meule, 2015). Il termine "food addiction" è stato però coniato solo nel 1956 da Theron Randolph per descrivere il consumo, simile a quello che si osserva nel disturbo da uso di sostanze, di alcuni alimenti come il mais, il frumento, il caffè, il latte, le uova e le patate (Randolph, 1956).

Rispetto a questa descrizione originale, oggi l'attenzione si è spostata verso gli alimenti processati ricchi di zuccheri e grassi che, secondo i sostenitori della modello del food addiction, avrebbero proprietà che favoriscono lo sviluppo della dipendenza in alcuni soggetti vulnerabili (Schulte, Avena, \& Gearhardt, 2015). Più recentemente è stato proposto che questi alimenti potrebbero essere implicati nello sviluppo dell'epidemia globale dell'obesità (Leigh \& Morris, 2018) e di alcuni disturbi dell'alimentazione che si caratterizzano per la presenza di ricorrenti episodi di abbuffata (Treasure, Leslie, Chami, \& Fernandez-Aranda, 2018).

Questa ipotesi ha portato i sostenitori del modello del food addiction a raccomandare alla politica sanitaria di emanare leggi per regolare la disponibilità dei cibi considerati "additivi" e di identificare e trattare le persone che potrebbero soffrire di dipendenza da cibo (Carter et al., 2016).

La proposta dell'esistenza della food addiction ha stimolato numerose ricerche e discussioni nella letteratura scientifica, ma i dati disponibili sono controversi e l'opinione di clinici e ricercatori è divisa. Scopo di questo articolo è fornire un aggiornamento sintetico della validità e dell'utilità clinica del modello del food addiction applicato in particolare ai disturbi dell'alimentazione e all'obesità.

\section{Qual è il modello del food addiction}

Secondo il modello del food addiction gli episodi di alimentazione in eccesso sono il risultato di un processo fisiologico sottostante, equivalente a quello responsabile dell'alcolismo. Le persone con food addiction sarebbero biologicamente vulnerabili a certi alimenti (tipicamente zuccheri e grassi) e, diventando dipendenti da essi, sarebbero incapaci di controllare la quantità della loro assunzione. Poiché la vulnerabilità è determina biologicamente, ne consegue che chi è affetto dalla "malattia" food addiction non può mai guarire, ma piuttosto deve imparare ad accettarla e ad aggiustare la sua vita per poterla gestire.

Non c'è comunque un accordo tra i sostenitori del modello del food addiction, se esso sia una dipendenza da una sostanza, come il disturbo da uso di alcool, oppure una dipendenza comportamentale, come il disturbo da gioco d'azzardo, recentemente inserito nel Manuale Diagnostico e Statistico dei Disturbi Mentali (DSM-5) all'interno della categoria diagnostica dei disturbi da uso di sostanze (American Psychiatric Association, 2013). Alcuni autori, infatti, affermando che l'evidenza attuale dell'esistenza di cibi con proprietà tali da creare dipendenza sono scarse, hanno sostenuto l'esistenza di un comportamento alimentare additivo, e hanno proposto di usare il termine "eating addiction" (Hebebrand et al., 2014).

\section{Valutare la food addiction: la Yale Food Addiction Scale}

La Yale Food Addiction Scale, o YFAS, è una scala self-report sviluppata per identificare le persone che hanno più probabilità di esibire dei marcatori di dipendenza nei confronti del consumo di cibi ricchi di grassi e zuccheri. Lo 
strumento è basato su 25 item, che valutano la presenza dei sette criteri sintomatici per la dipendenza da sostanze, definiti dal DSM-IV-TR: tolleranza, astinenza, perdita di controllo, desiderio e fallimenti, tempo speso, rinuncia ad attività sociali, lavorative o ricreative importanti, uso continuato nonostante le conseguenze persistenti.

Due dei 25 item valutano la presenza di danno clinico significativo, o disagio, derivante dall'alimentazione in eccesso (Gearhardt, Corbin, \& Brownell, 2009). Una diagnosi dicotomica di dipendenza da cibo si applica quando sono presenti almeno tre criteri sintomatici e un danno clinico significativo. In alternativa, può essere calcolato un punteggio dei sintomi da 1 a 7 per misurare la gravità della sintomatologia presente.

Una recente revisione, che ha valutato 40 studi sperimentali sugli esseri umani pubblicati dopo il 2009, ha concluso che il punto non ancora chiarito è se gli individui che soddisfano i criteri YFAS per la diagnosi di food addiction siano veramente dipendenti dal cibo e se sperimentino un danno clinico significativo nelle aree del benessere psicologico e della qualità della vita, come accade nei disturbi da uso di sostanze clinicamente riconosciuti (Long, Blundell, \& Finlayson, 2015).

La revisione ha evidenziato che una diagnosi positiva di food addiction determinata dalla YFAS si associa positivamente con l'indice di massa corporea (BMI) e gli episodi di abbuffata (pur con qualche eccezione), mentre i dati disponibili sulla capacità della YFAS di valutare una relazione tra danno clinico significativo e assunzione di cibo sono scarsi.

Gli autori della revisione sottolineano inoltre che avere valutato la cosiddetta food addcition con la sola YFAS introduce la possibilità di un argomento circolare. Vale a dire: alla domanda: "Perché questa persona è dipendente da cibo?", la risposta è "Perché ha un punteggio elevato alla YFAS". Ma alla domanda "Perché questa persona ha punteggio elevato alla YFAS?", la risposta è "Perché è dipendente dal cibo". Infine, sottolineano perché definire la cosiddetta food addiction con gli stessi criteri del disturbo da uso di sostanze è controverso: a differenza delle sostanze stupefacenti o del gioco d'azzardo, infatti, il cibo e il mangiare sono essenziali per la sopravvivenza degli esseri umani (Long et al., 2015).

\section{Episodi di abbuffata e uso di sostanze}

Nell'ambito dell'alimentazione, il comportamento che più di altri ha fatto ipotizzare una dipendenza dal cibo è sicuramente l'abbuffata. Indubbiamente l'episodio di abbuffa- ta e l'uso di sostanze hanno molte caratteristiche in comune, ma le differenze sono altrettanto rilevanti e il legame tra i due comportamenti è non specifico.

\section{Similitudini}

Le principali similitudini tra gli episodi di abbuffata e il disturbo da uso di sostanze sono (Fairburn, 2013):

- Il craving (cioè il desiderio intenso o la spinta) ad adottare il comportamento

- La sensazione di perdita di controllo nei confronti del comportamento

- La preoccupazione nei confronti del comportamento

- L'uso del comportamento per mitigare la tensione e le emozioni negative

- La negazione della gravità del problema

- Il tentativo di mantenere il problema segreto

- La persistenza del comportamento nonostante i suoi effetti dannosi

- I frequenti e ripetuti insuccessi nel tentativo di sospendere il comportamento.

Come sottolineato da Fairburn (2013) queste similitudini sono parziali e, sebbene alcune siano rilevanti per il trattamento (per esempio l'uso del comportamento per alleviare la tensione e le emozioni negative), non significa che i meccanismi sottostanti ai comportamenti legati all'assunzione di cibo e di sostanze stupefacenti siano gli stessi. Focalizzarsi esclusivamente sulle similitudini, inoltre, non permette di fare emergere alcune importanti differenze che potrebbero essere fondamentali per capire i meccanismi sottostanti o ottimizzare il trattamento.

\section{Differenze}

Come è stato accennato, esistono importanti differenze tra gli episodi di abbuffata e il disturbo da uso di sostanze.

1. Gli episodi di abbuffata non implicano il consumo di particolari classi di alimenti (Wilson, 2010). Se l'episodio di abbuffata fosse una forma di dipendenza dovrebbe caratterizzarsi dal desiderio e dal consumo di specifici alimenti. Questo però non accade nelle persone con bulimia nervosa e disturbo da binge-eating, dove l'aspetto caratteristico dell'episodio di abbuffata, come specificato dai criteri diagnostici del DSM-5 (American Psychiatric Association, 2013), è la quantità di cibo assunta non quello che viene mangiato.

2. Gli individui con disturbi dell'alimentazione hanno una continua spinta ad evitare l'episodio di abbuffata. $\mathrm{Al}$ 
contrario, una delle maggiori difficoltà nel trattamento del disturbo da uso di sostanze è motivare gli individui ad evitare l'uso della sostanza (Fairburn, 2013).

3. Gli individui che si abbuffano spesso adottano una dieta ferrea per perdere peso, la quale aumenta la vulnerabilità ad avere episodi di abbuffata (Fairburn, 2013). Al contrario, gli individui con disturbo da uso di sostanze e di alcool non sono vulnerabili all'abuso della sostanza quando cercano di non assumerla (Benton, 2010).

4. Il disturbo da binge-eating è causato dall' interazione di numerosi fattori di rischio sociali e psicologici, non esclusivamente relati alla nutrizione (Fairburn et al., 1998). Questo indicherebbe che gli episodi di abbuffata sarebbero più una modalità usata per modulare le emozioni negative e lo stress piuttosto che la conseguenza della dipendenza nei confronti del cibo.

\section{Relazione non specifica}

La relazione tra episodi di abbuffata e uso di sostanze non è specifica (Wilson, 2010): infatti, sebbene il tasso di uso di sostanze e di alcool negli individui che si abbuffano sia più elevato rispetto alla popolazione, esso è però simile a quello di individui con altri disturbi psichiatrici (Kushner, Sher, \& Beitman, 1990). Allo stesso modo, il tasso di episodi di abbuffata è più elevato negli individui con disturbo da uso di alcool e sostanze, ma è simile a quello degli individui con altri disturbi psichiatrici (Fairburn, 2013).

Anche la presenza di uso di sostanze nei familiari degli individui che si abbuffano non è superiore a quella osservata in altri disturbi psichiatrici (Fairburn, 2013). Infine, sebbene sia stato osservato che i problemi alimentari precedano l'abuso di alcool (insorgono cioè a un'età più precoce), i dati sul trattamento indicano che i pazienti che interrompono gli episodi di abbuffata non li sostituiscono con l'abuso di alcool (Karacic et al., 2011).

\section{Alcuni dati neurobiologici}

Alcuni studiosi, con l'obiettivo di chiarire il legame tra assunzione di cibo in eccesso e l'uso di sostanze, hanno condotto studi neurobiologici di confronto tra soggetti con obesità e normopeso, ma i risultati appaiono inconsistenti.

Nel 2001 uno studio con la tomografia a emissione di positroni (PET), in un gruppo di soggetti con obesità, ha dimostrato la presenza di una riduzione dei recettori striatali D2R della dopamina, negativamente correlato con l'IMC (Wang et al., 2001): questo dato potrebbe indicare uno stato di deficit di ricompensa, la quale favorirebbe una maggiore assunzione di cibo per ottenere lo stesso livello di ricom- pensa percepito dai soggetti di peso normale. Il disegno dello studio, basato su osservazioni cross-sectional, non spiega però se i cambiamenti dei recettori siano una causa, o una conseguenza dell'obesità. Inoltre, studi successivi sui recettori D2R nei soggetti con obesità hanno prodotto risultati inconsistenti (Ziauddeen, Farooqi, \& Fletcher, 2012).

In modo simile, sebbene gli studi di neuroimaging che esplorano le risposte del cervello agli stimoli alimentari e relativi al cibo nelle persone di peso normale abbiano mostrato un'attivazione ampiamente coerente nel circuito della ricompensa (tra cui l'amigdala, l'insula e lo striato), i modelli che emergono dagli studi di confronto tra individui con obesità ed episodi di abbuffata e soggetti di controllo si caratterizzano per un'ampia variabilità e inconsistenza (Ziauddeen et al., 2012).

\section{Disturbi da uso di sostanze, obesità e disturbi dell'alimentazione}

\section{Diverso decorso temporale della ricaduta}

I disturbi da uso di sostanze, l'obesità e i disturbi dell'alimentazione hanno modalità di ricaduta uniformi, ma sorprendentemente diverse nel tempo (Wilson, 2010). Nei disturbi da uso di sostanze il tasso di ricaduta decelera dopo che è stata ottenuta l'astinenza iniziale: ne deriva che la quantità di tempo di astinenza accumulato può essere la variabile chiave, che modella la curva di recidiva. In altre parole, "l'astinenza genera l'astinenza". Infatti, se si considera il tasso di ricaduta dopo un anno dalla fine del trattamento dei disturbi da uso di nicotina, eroina e alcool, la maggior parte dei pazienti ricade precocemente dopo la conclusione del trattamento, ma poi il tasso di ricaduta decelera in modo molto significativo.

Accade il contrario nel trattamento comportamentale dell'obesità: infatti il tasso di perdita di peso iniziale è rapido, poi lentamente diminuisce e raggiunge il picco circa sei mesi dopo l'inizio del trattamento. Da questo punto in poi il peso è gradualmente recuperato. I disturbi dell'alimentazione trattati con la terapia cognitivo comportamentale, invece, mostrano un sostanziale mantenimento dei miglioramenti raggiunti (Wilson, 2010).

Queste osservazioni sottolineano le differenze fondamentali tra obesità e disturbi dell'alimentazione, che non sono in linea con quanto proposto dal modello del food addiction. Suggeriscono inoltre l'esistenza di meccanismi diversi (biologici, psicologici o sociali), alla base del mantenimento, della remissione e della ricaduta nel disturbo da uso di sostanze, piuttosto che nell'obesità, o nei disturbi dell'alimentazione (Wilson, 2010). 


\section{Meccanismi di mantenimento dell'episodio di abbuffata nei disturbi dell'alimentazione}

La teoria cognitivo comportamentale transdiagnostica, che ha attualmente il maggiore supporto empirico, sostiene che il nucleo psicopatologico centrale dei disturbi dell'alimentazione è l'eccessiva valutazione del peso e della forma del corpo, cioè la valutazione di sé influenzata in modo predominante dal peso, della forma del corpo e dal loro controllo (Fairburn, 2008). Da questa psicopatologia nucleare deriva la maggior parte delle caratteristiche dei disturbi dell'alimentazione: tra queste l'adozione di regole dietetiche estreme e rigide che, secondo la teoria, svolgono un ruolo chiave nello sviluppo e nel mantenimento degli episodi di abbuffata.

Le persone con disturbi dell'alimentazione hanno infatti la tendenza a reagire in modo negativo ed estremo (spesso dicotomico) alla quasi inevitabile rottura di queste regole: anche una piccola trasgressione tende a essere interpretata come la prova di scarso autocontrollo e debolezza personale. La risposta a questa percepita mancanza di autocontrollo è un temporaneo abbandono dello sforzo di restringere l'alimentazione, che sfocia nell'episodio di abbuffata. Quest'ultimo mantiene la psicopatologia nucleare, intensificando le preoccupazioni di essere incapaci di controllare il peso, la forma del corpo e l'alimentazione, incoraggia un'ulteriore restrizione dietetica e aumenta così il rischio di ulteriori episodi di abbuffata.

Tre ulteriori processi contribuiscono a mantenere gli episodi di abbuffata:

1. Le difficoltà della vita e i cambiamenti emotivi associati aumentano la probabilità che le persone con disturbi dell'alimentazione rompano le loro regole dietetiche rigide ed estreme.

2. Dal momento che l'episodio di abbuffata migliora temporaneamente il tono dell'umore e distrae dai problemi, esso può diventare un mezzo disfunzionale per far fronte a queste difficoltà (è un meccanismo spesso implicato nel mantenimento degli episodi di abbuffata negli individui con disturbi da binge-eating, che non adottano regole dietetiche estreme e rigide).

3. Se l'episodio di abbuffata è seguito dal vomito autoindotto, o dall'uso di altri comportamenti di compenso, questi tendono a mantenere gli episodi di abbuffata, perché le persone hanno la convinzione che tali comportamenti siano efficaci nel prevenire l'assorbimento di calorie e, di conseguenza, allentano il controllo dell'alimentazione perché viene meno un importante deterrente dell'alimentazione in eccesso (cioè la paura d'ingrassare).

\section{Meccanismi di mantenimento dell'alimentazione edonica nell'obesità}

Secondo alcuni autori, l'uso del modello del food addiction, oltre a stigmatizzare l'individuo (nel DSM-5 si usa il termine disturbo da uso di sostanze per evitare che accada questo), è una forma di medicalizzazione non necessaria dell'alimentazione eccessiva edonica (Finlayson, 2017). Negli individui con obesità che mangiano in eccesso per gratificarsi non è, infatti, necessario adottare il modello del food addiction per spiegare il loro comportamento; esso può derivare dal "processo di apprendimento di abitudini" non innate, che si sviluppa attraverso processi edonici normali (non patologici), in un ambiente alimentare denso di energia e culturalmente permissivo (Finlayson, 2017).

Due processi sembrano particolarmente implicati nell'acquisizione e nella persistenza di abitudini alimentari disfunzionali (Graybiel, 2008). Il primo è l'apprendimento basato sull'esito dell'azione (per esempio il piacere che comporta l'assunzione di alcuni cibi). Chiamata anche condizionamento strumentale o operante, questa forma di apprendimento coinvolge specifiche vie neurali come l'amigdala, lo striato ventrale (il nucleo accumbens) e la corteccia orbito frontale.

Il secondo è quello dello stimolo-risposta, chiamato anche condizionamento classico, che si sviluppa quando uno stimolo non condizionato (per esempio un'emozione, un momento della giornata, o un'attività) è associato ripetutamente con l'assunzione di determinati alimenti e coinvolge altre strutture neurali: lo striato dorso laterale (il caudato/ putamen) e la corteccia prefrontale dorso laterale.

Va anche sottolineato che, sebbene alcuni cibi attivino attività neurali comuni a quelle delle sostanze stupefacenti, nessuna evidenza ha ancora dimostrato che esista una sensibilizzazione neurale al cibo (Finlayson, 2017). Il meccanismo sarebbe addirittura contrario: la stimolazione intensa determinata dalle sostanze stupefacenti, che eccede di molto quella ottenuta da qualsiasi cibo, sembra essere la causa della disfunzione del sistema della ricompensa naturale (inclusa la sensibilizzazione dei neuroni della dopamina mesolimbica coinvolti nel processo del desiderio), piuttosto che la mera attivazione delle vie del desiderio. Ecco perché la dipendenza da sostanze diventa così compulsiva e persistente, indipendentemente dal piacere e dai danni che la loro assunzione comporta (Robinson \& Berridge, 1993). Nello stesso tempo, questo significa che un alimento in grado di attivare il sistema della ricompensa non può essere classificato automaticamente come sostanza che crea dipendenza (Finlayson, 2017). 


\section{Implicazioni per il trattamento}

Dal momento che la dipendenza è considerata una malattia del cervello (Leshner, 1997), è stato proposto di inserire la food addiction e l'obesità nel DSM-5, ma la proposta e stata scoraggiata (Marcus \& Wildes, 2009) e non accettata (American Psychiatric Association, 2013). L'obesità oggi è infatti considerata un disturbo ereditabile neurocomportamentale, molto sensibile alle condizioni ambientali (O’Rahilly \& Farooqi, 2008).

Allo stesso modo, i disturbi dell'alimentazione che hanno tra le loro espressioni psicopatologiche gli episodi di abbuffata (cioè la bulimia nervosa e il disturbo da binge-eating), pur essendo classificati dal DSM-5 come malattie mentali, non sono stati inclusi nella categoria diagnostica del disturbo da uso di sostanze, ma in quella dei disturbi della nutrizione e dell'alimentazione perché, come chiarito in precedenza, derivano dall'interazione di numerosi fattori di rischio sociali e psicologici e non da fattori esclusivamente relati alla nutrizione; inoltre presentano una psicopatologia specifica (per esempio l'eccessiva valutazione del peso e della forma del corpo), assente nei disturbi da uso di sostanze.

Il considerare l'alimentazione eccessiva e incontrollata come una dipendenza avrebbe ripercussioni inevitabili sulla terapia. Il trattamento non farmacologico della food addiction, infatti, dovrebbe essere basato sull'approccio che Alcolisti Anonimi e altri gruppi simili applicano per aiutare le persone con problemi di alcol: il cosiddetto programma dei 12 passi.

Lapproccio, come mostrato nella Tabella 1, differisce in modo sostanziale dalla terapia cognitivo comportamentale dei disturbi dell'alimentazione (CBT-ED), raccomandata dalle linee guida del National Institute for Care and Health (NICE) (2017) per il trattamento di tutte le forme di disturbi dell'alimentazione negli adulti e negli adolescenti. Queste linee guida, che riflettono le nuove evidenze sostanziali emerse nel decennio precedente, si sono concentrate sui risultati di studi clinici controllati, ma hanno anche considerato l'esperienza degli utenti, dei familiari, dei ricercatori e dei medici.

Inoltre, per quanto riguarda il trattamento non farmacologico dell'obesità, non esistono dati sull'efficacia a lungo termine dei trattamenti basati sull'approccio di 12 passi. Diversi sono invece i riscontri della terapia cognitivo comportamentale, che aiuta il paziente ad affrontare gli ostacoli ambientali, cognitivi ed emotivi della perdita e del mantenimento del peso (Dalle Grave, Sartirana, El Ghoch, \&

Tabella 1. Differenze tra l'approccio basato sui 12 passi e la terapia cognitivo comportamentale dei disturbi dell'alimentazione (CBT-ED)

\begin{tabular}{l}
12 passi \\
\hline Il disturbo è una malattia che non guarisce mai \\
\hline È fondamentale l'astinenza immediata dagli episodi di \\
abbuffata (i partecipanti identificati come astinenti sono \\
lodati, mentre a quelli non astinenti è data poca possibilità \\
di parlare - a volte è chiesto loro di lasciare il gruppo) \\
\hline Per raggiungere l'astinenza è necessario astenersi anche \\
dall'assunzione dei cibi considerati tossici che stimolano gli \\
episodi di abbuffata
\end{tabular}

Si è in controllo o fuori dal controllo; i cibi sono salutari o tossici; si è astinenti o non astinenti

\section{CBT-E}

La guarigione completa è raggiunta dalla maggior parte delle persone (dato confermato da studi a lungo termine di follow-up della bulimia nervosa e del disturbo da bingeeating)

L'immediata cessazione degli episodi di abbuffata non è realistica (la sospensione delle abbuffate è raggiunta gradualmente con l'interruzione dei meccanismi di mantenimento identificati)

L'evitamento dei cibi va eliminato non incoraggiato (i dati clinici e della ricerca indicano che è il tentativo di non mangiare questi cibi che favorisce gli episodi di abbuffata)

La modalità di pensiero tutto o nulla è un problema che va affrontato (il trattamento incoraggia a considerare una singola perdita di controllo nei confronti dell'alimentazione come una scivolata - lapse - e non una ricaduta - relapse - da cui si può imparare per affrontare con successo una situazione ad alto rischio simile in futuro. Le modalità di pensiero tutto o nulla sono implicate nel mantenimento degli episodi di abbuffata) 
Calugi, 2017): i dati infatti indicano la sua efficacia nell'indurre una duratura perdita di peso, fino a 8 anni dall'inizio del trattamento (Look Ahead Research Group, 2014).

Dal momento che la food addiction è considerata una malattia del cervello, il suo trattamento dovrebbe essere risolutivo ricorrendo a famaci usati nel disturbo da uso di sostanze. Un farmaco recentemente approvato in Italia (naltrexolone cloroidrato + buproprione cloroidrato), che utilizza principi attivi per il trattamento del disturbo da uso di oppiacei e di nicotina, è stato infatti messo alla prova in quattro studi, a confronto con placebo, ma gli effetti sono stati limitati: il calo ponderale ottenuto era compreso tra $3,7 \%$ e $8,1 \%$, rispetto all' $1,3 \%$ e al $4,1 \%$ del placebo (Ali, Shukla, \& Aronne, 2016).

La complessità dei meccanismi che regolano l'introito di cibo, il dispendio energetico e il mantenimento dell'obesità rappresenta, infatti, una sfida per lo sviluppo di farmaci efficaci per la perdita di peso, che siamo molto lontani da poter vincere.

\section{Conclusioni}

Nonostante la presenza di molte similitudini tra gli episodi di abbuffata osservati nelle persone con disturbi dell'alimentazione e il disturbo da uso di sostanze, esistono fondamentali differenze tra i due disturbi che riguardano la psicopatologia, l'epidemiologia e i fattori di rischio. Di particolare importanza e preoccupazione è la possibile adozione di un trattamento basato sul modello della food addiction nei disturbi dell'alimentazione perché, avendo implicazioni terapeutiche che contraddicono esplicitamente la CBT-ED, il trattamento attualmente raccomandato per i pazienti adulti e adolescenti affetti da disturbi dell'alimentazione (NICE, 2017) potrebbe allontanare le persone da terapie di provata efficacia.

Per quanto riguarda l'obesità, sebbene la ricerca neurobiologica abbia rivelato elementi comuni nei processi di ricompensa cerebrale, questo non significa che un alimento in grado di attivare il sistema della ricompensa possa essere classificato come sostanza che crea dipendenza. Infatti, l'alimentazione in eccesso riportata da alcune persone con obesità può essere spiegata attraverso l'acquisizione di abitudini determinate dall'attivazione di processi edonici normali (non patologici), in un ambiente alimentare denso di energia e culturalmente permissivo. Inoltre, tra l'obesità e il disturbo da uso di sostanze esistono importanti differenze, come il decorso temporale della ricaduta e gli esiti del trattamento.

Anche il sostenere il concetto di eating addiction appare problematico perché si corre il rischio di usare il termi- ne "addiction" per qualsiasi comportamento ripetitivo e abitudinario, come guardare la televisione, praticare l'attività sessuale, fare shopping. Quando, infatti, la parola addiction è usata in modo allargato e omnicomprensivo, la maggior parte delle persone potrebbe dire di essere dipendente da qualcosa (Wilson, 2010).

È importante comunque sottolineare che, anche se si esclude il modello del food addiction nella genesi e nel mantenimento dei disturbi dell'alimentazione e dell'obesità, è raccomandabile incoraggiare l'implementazione di interventi preventivi di salute pubblica, per creare un ambiente che permetta alla maggior parte degli individui di adottare un'alimentazione salutare e uno stile di vita attivo.

\section{Bibliografia}

Ali, K. F., Shukla, A. P., \& Aronne, L. J. (2016). BupropionSR plus naltrexone-SR for the treatment of mild-tomoderate obesity. Expert Rev Clin Pharmacol, 9(1), 2734. doi:10.1586/17512433.2016.1100072

American Psychiatric Association. (2013). Diagnostic and Statistical Manual of Mental Disorders (DSM-5). Washinton, DC: American Psychiatric Association.

Benton, D. (2010). The plausibility of sugar addiction and its role in obesity and eating disorders. Clin Nutr, 29(3), 288-303. doi:10.1016/j.clnu.2009.12.001

Carter, A., Hendrikse, J., Lee, N., Yucel, M., Verdejo-Garcia, A., Andrews, Z., \& Hall, W. (2016). The Neurobiology of "Food Addiction" and Its Implications for Obesity Treatment and Policy. Annu Rev Nutr, 36, 105-128. doi:10.1146/annurev-nutr-071715-050909

Dalle Grave, R., Sartirana, M., El Ghoch, M., \& Calugi, S. (2017). Personalized multistep cognitive behavioral therapy for obesity. Diabetes, Metabolic Syndrome and Obesity: Targets and Therapy, 10, 195-206. doi:10.2147/ DMSO.S139496

Fairburn, C. G. (2008). Cognitive behavior therapy and eating disorders. New York: Guilford Press.

Fairburn, C. G. (2013). Overcomimg bing eating (second edition). New York: Guilford Press.

Fairburn, C. G., Doll, H. A., Welch, S. L., Hay, P. J., Davies, B. A., \& O'Connor, M. E. (1998). Risk factors for binge eating disorder: a community-based, case-control study. Archives of General Psychiatry, 55(5), 425-432.

Finlayson, G. (2017). Food addiction and obesity: unnecessary medicalization of hedonic overeating. Nat Rev Endocrinol, 13(8), 493-498. doi:10.1038/ nrendo.2017.61 
Gearhardt, A. N., Corbin, W. R., \& Brownell, K. D. (2009). Preliminary validation of the Yale Food Addiction Scale. Appetite, 52(2), 430-436. doi:10.1016/j. appet.2008.12.003

Graybiel, A. M. (2008). Habits, rituals, and the evaluative brain. Annu Rev Neurosci, 31, 359-387. doi:10.1146/ annurev.neuro.29.051605.112851

Hebebrand, J., Albayrak, O., Adan, R., Antel, J., Dieguez, C., de Jong, J., ... Dickson, S. L. (2014). "Eating addiction”, rather than "food addiction", better captures addictivelike eating behavior. Neurosci Biobehav Rev, 47, 295306. doi:10.1016/j.neubiorev.2014.08.016

Karacic, M., Wales, J. A., Arcelus, J., Palmer, R. L., Cooper, Z., \& Fairburn, C. G. (2011). Changes in alcohol intake in response to transdiagnostic cognitive behaviour therapy for eating disorders. Behaviour Research and Therapy, 49(9), 573-577. doi:10.1016/j.brat.2011.05.011

Kushner, M. G., Sher, K. J., \& Beitman, B. D. (1990). The relation between alcohol problems and the anxiety disorders. Am J Psychiatry, 147(6), 685-695. doi:10.1176/ ajp.147.6.685

Leigh, S. J., \& Morris, M. J. (2018). The role of reward circuitry and food addiction in the obesity epidemic: An update. Biol Psychol, 131, 31-42. doi:10.1016/j. biopsycho.2016.12.013

Leshner, A. I. (1997). Addiction is a brain disease, and it matters. Science, 278(5335), 45-47.

Long, C. G., Blundell, J. E., \& Finlayson, G. (2015). A Systematic Review of the Application And Correlates of YFAS-Diagnosed 'Food Addiction' in Humans: Are Eating-Related 'Addictions' a Cause for Concern or Empty Concepts? Obes Facts, 8(6), 386-401. doi:10.1159/000442403

Look Ahead Research Group. (2014). Eight-year weight losses with an intensive lifestyle intervention: the look AHEAD study. Obesity (Silver Spring), 22(1), 5-13. doi:10.1002/oby.20662

Marcus, M. D., \& Wildes, J. E. (2009). Obesity: is it a mental disorder? Int J Eat Disord, 42(8), 739-753. doi:10.1002/ eat. 20725
Meule, A. (2015). Back by Popular Demand: A Narrative Review on the History of Food Addiction Research. Yale J Biol Med, 88(3), 295-302.

NICE. (2017). Eating disorders: recognition and treatment (NG69). London: National Institute for Clinical Excellence Retrieved from https://www.nice.org.uk/ guidance/ng69.

O’Rahilly, S., \& Farooqi, I. S. (2008). Human obesity: a heritable neurobehavioral disorder that is highly sensitive to environmental conditions. Diabetes, 57(11), 2905-2910. doi:10.2337/db08-0210

Randolph, T. G. (1956). The descriptive features of food addiction: Addictive eating and drinking. Q J Stud Alcohol, 17, 198-224.

Robinson, T. E., \& Berridge, K. C. (1993). The neural basis of drug craving: an incentive-sensitization theory of addiction. Brain Res Brain Res Rev, 18(3), 247-291.

Schulte, E. M., Avena, N. M., \& Gearhardt, A. N. (2015). Which foods may be addictive? The roles of processing, fat content, and glycemic load. PLoS One, 10(2), e0117959. doi:10.1371/journal.pone.0117959

Treasure, J., Leslie, M., Chami, R., \& Fernandez-Aranda, F. (2018). Are trans diagnostic models of eating disorders fit for purpose? A consideration of the evidence for food addiction. Eur Eat Disord Rev. doi:10.1002/erv.2578

Wang, G. J., Volkow, N. D., Logan, J., Pappas, N. R., Wong, C. T., Zhu, W., ... Fowler, J. S. (2001). Brain dopamine and obesity. Lancet, 357(9253), 354-357.

Wilson, G. T. (2010). Eating disorders, obesity and addiction. Eur Eat Disord Rev, 18(5), 341-351. doi:10.1002/erv.1048

Ziauddeen, H., Farooqi, I. S., \& Fletcher, P. C. (2012). Obesity and the brain: how convincing is the addiction model? Nat Rev Neurosci, 13(4), 279-286. doi:10.1038/ nrn3212 